\title{
Erratum to: Taxonomic dissolution of Sarcostemma (Apocynaceae: Asclepiadoideae)
}

\author{
Ulrich Meve $^{1} \&$ Sigrid Liede-Schumann ${ }^{1}$
}

Summary. Due to a production error, an out-of-date (uncorrected) pdf of this paper was uploaded onto Springerlink, and used for the print version of Kew Bulletin 67 (4): 751 - 758 (10.1007/s12225-012-9384-2). Consequently the authority for Cynanchum viminale (L.) Bassi and its subspecies was incorrect. These names are now listed again below with the correct authority.

Cynanchum viminale (L.) Bassi (1768: 17) subsp. viminale

NOTES. The protologue of Cynanchum (Euphorbia) viminale (Linnaeus 1753) refers to an illustration of a plant from Egypt (Felfel Tavil) as the type and states explicitly 'Habitat in Africae maritimis'.

Cynanchum viminale (L.) Bassi subsp. australe (R. Br.) Meve Ẽ Liede, comb. nov.

http://www.ipni.org/urn:lsid:ipni.org:names:77120428-1

Sarcostemma australe R. Br., Prodr.: 463 (Brown 1810). Type: Australia, South Australia, Bird Island, Petral Bay, Isle St. Francis, 3 Feb. 1802, R. Brown (Iter Australiense 2872) (lectotype BM!, designated by Forster (1992)).

Sarcostemma viminale subsp. australe (R. Br.) P. I. Forst., Austral. Syst. Bot. 5: 64 (Forster 1992).

Cynanchum viminale (L.) Bassi subsp. brunonianum (Wight Ẽ Arn.) Meve Ẽ Liede, comb. nov.

http://www.ipni.org/urn:lsid:ipni.org:names:77120429-1

Sarcostemma brunonianum Wight \& Arn., in Wight, Contr. Bot. India: 59 (1834). Type: India, ['Peninsula Ind. Orientalis'], Cody Cally, village of Autcherroccum, Herb. Wright 1557 (lectotype G-DC! (No. G00301010), designated here; isolectotypes E, G-DC! (No. G00136527)).

Sarcostemma viminale (L.) R. Br. subsp. brunonianum (Wight \& Arn.) P. I. Forst. (Forster 1992: 63).
Cynanchum viminale (L.) Bassi subsp. orangeanum (Liede $\mathcal{E}$ Meve) Liede $\mathcal{E}$ Meve, comb. nov.

http:/ /www.ipni.org/urn:lsid:ipni.org:names:77120430-1

Sarcostemma viminale subsp. orangeanum Liede \& Meve, Bot. J. Linn. Soc. 112: 7 (1993). Type: South Africa, Northern Cape, 11.4 km E Kuruman, Liede E̋ Meve 579 (holotype $\mathrm{K}$ !; isotype MSUN!).

Cynanchum viminale (L.) Bassi subsp. stocksii (Hook.f.) Meve $\mathcal{E}$ Liede, comb. nov.

http:/ /www.ipni.org/urn:lsid:ipni.org:names:77120431-1

Sarcostemma stocksii Hook. f., Fl. Brit. India 4: 27 (Hooker 1883). Type: Pakistan, Sind, Stocks 509 (holotype K!). Sarcostemma viminale subsp. stocksii (Hook. f.) Ali (1983: 31).

Cynanchum viminale (L.) Bassi subsp. thunbergii (G. Don) Liede Ẽ Meve, comb. nov.

http:/ /www.ipni.org/urn:lsid:ipni.org:names:77120432-1

Sarcostemma thunbergii G. Don, Gen. Hist. 4: 156 (1838). Type: South Africa, Cape province, Worcester, Karoo Botanic Garden, Bayer 68 (neotype NBG!, designated by Liede \& Meve (1993).

Sarcostemma viminale subsp. thunbergii (G. Don) Liede \& Meve (1993: 10).

Cynanchum viminale (L.) Bassi subsp. welwitschii (Hiern) Meve E Liede, comb. et stat. nov.

The online version of the original article can be found at http://dx.doi.org/10.1007/s12225-012-9384-2

Published online 26 March 2013

1 Department of Plant Systematics, University of Bayreuth, 95440 Bayreuth, Germany. e-mail: ulrich.meve@uni-bayreuth.de 
http://www.ipni.org/urn:lsid:ipni.org:names:77120433-1

Sarcostemma welwitschii Hiern, Cat. Afr. Pl. 1: 689 (1898). Type: Angola, Huilla, base of Morro de Lopollo, Welwitsch 4261 (holotype BM!; isotypes BR!, C).

\section{References}

Ali, S. I. (1983). Asclepiadaceae, No. 150. In: E. Nasir \& S. I. Ali (eds), Flora of Pakistan, pp. 1 - 65. Shamim Printing Press, Karachi.

Bassi, F. (1768). Novae plantarum species. Bononiensi Sci. Inst. Acad. Comment. 6 (Opusc.): 13 - 20.

Brown, R. (1810). Asclepiadeae. In: Prodromus Florae Novae Hollandiae, pp. 458 - 464. J. Johnson \& Co., London.

Don, G. (1838). Asclepiadeae. In: General History of the Dichlamydeous Plants 4, pp. 106 - 166. J. G. \& F. Rivington, London.
Forster, P. I. (1992). A taxonomic revision of Sarcostemma R. Br. Subgenus Sarcostemma (Asclepiadaceae: Asclepiadeae) in Australia. Austral. Syst. Bot. 5: $53-70$.

Hiern, W. P. (1898). Asclepiadaceae. In: Catalogue of the African Plants collected by Dr. Friedrich Welwitsch, dicotyledons, part 3, pp. 676 - 698. Printed by order of the Trustees, London.

Hooker, J. D. (1883). Asclepiadaceae to Amaranthaceae. The Flora of British India 4: 1 - 78. L. Reeve \& Co., London (Repr. Singh, Dehra Dun).

Liede, S. \& Meve, U. (1993). Towards an understanding of the Sarcostemma viminale (Asclepiadaceae) complex. Bot. J. Linn. Soc. 112: 1 - 15.

Linnaeus, C. (1753). Euphorbia viminalis. Species Plantarum. P. 452. L. Salvii, Holmiae (Stockholm).

Wight, R. (1834). Asclepiadae Indicae. In R. Wight (ed.), Contributions to the Botany of India, pp. $29-67$. Parbury, Allen \& Co., London. 